\title{
The perception of pause in question-answer pairs
}

\author{
VALENTINA D'URSO and VANDA ZAMMUNER \\ University of Padua, Padua, Italy
}

\begin{abstract}
The hypothesis that the duration of interturn pauses between questions and answers in verbal exchange is predictable was tested in two experiments. In Experiment 1, subjects estimated the lengths of 3-and 7-sec gaps inserted between questions and answers in their own language and in a language they did not understand; they also estimated gaps inserted into a sequence of white noise. In Experiment 2, subjects conducted a telephone interview with a person who was instructed to intentionally delay beginning to answer some of the questions posed; the dependent variable was the amount of time the interviewer waited for the answer before spontaneously taking another turn. In both studies, the amount of pause perceived or found acceptable between a question and an answer varied as a function of linguistic factors or encyclopedic knowledge. The results are interpreted as reflecting a rule in language use that relates the rhythm of turn-taking to the amount of mental work plausibly needed to answer a question.
\end{abstract}

In normal conversations, the rhythm of turn-taking is a highly relevant feature of linguistic behavior. This issue, though investigated to some extent by conversation analysts using ethnomethodological procedures, is still in need of serious and detailed experimental study. In particular, we believe that in order to account fully for the phenomenon, cognitive aspects, including the content of what is said, must be considered. This will require the development of a model that takes into account the mental processes involved in turn-taking and that can explain its temporal pattern. Such a model might initially be elaborated through observation of how what is said in one conversational turn affects what is said in the next, or adjacent, turn. We assume here that such a focus is motivated, since it is plausible that the temporal pace of dialogue is limited or otherwise governed largely by inferences made about the contents of mental processes.

In the present study, we will be mainly concerned with how the content of the first of two adjacent conversational turns affects the length of a transition accepted before the second turn. This issue is especially relevent for understanding the temporal interval that separates questions and answers. The measures of interest here are the real and subjective durations of this interval. The main hypothesis we test is that these vary with the complexity of the discourse content.

This hypothesis is based on the more general idea that the temporal pace of a dialogue will be sensitive to the cognitive needs of language users as perceived by the conversational participants themselves. In this framework, pauses and gaps between turns in the flow of discourse are assumed to be expected and tolerated by a speakerlistener to the extent that the current content motivates them. The length of a transition will be processed along

Correspondence may be addressed to Vanda Zammuner, Dipartimento di Psicologia, Via Beato Pellegrino 26, Università degli Studi di Padova, 35137 Padua, Italy. with the message itself (cf. Butterworth, 1980; GoldmanEisler, 1968). In general, content complexity should help determine gap duration because it is proportional to the amount of mental work required to adequately process information carried by an utterance and to connect a following turn to it.

To test the hypothesis, we performed two experiments. In Experiment 1, we investigated how subjects perceive the duration of gaps inserted into auditory stimulus material of different kinds. Here, two predictions were made: (1) that the perceived duration of a gap inserted into an auditory signal will vary as a function of the type of material into which it is placed (Fraisse, 1978); (2) that, when a gap occurs between two conversational turns identifiable as an adjacency pair consisting of a question and answer, the easier the question is, the longer will be the gap perceived.

In Experiment 2, we investigated how soon a speaker will retake the floor when presented with a lack of feedback - that is, how a speaker copes with pause left between turns when conversing with another person. This was evaluated according to the length of the period during which a subject waited for an answer before intervening, after having asked another person particular questions.

\section{EXPERIMENT 1}


year class?" Examples of general knowledge questions included "What is the capital of Iceland?" and "Do rabbits live in Australia?" Presentation of the different kinds of material was blocked and counterbalanced across subjects tested.

Subjects, task, and procedure. The subjects listened to each sequence and immediately evaluated the duration of the gap by adjusting a chronometer whose time setting they could see. A total of 20 subjects, who were Italian university students unable to understand ancient Greek, evaluated the duration of each sequence.

\section{Results}

The data were analyzed using two between-subjects ANOVAs. An analysis including materials (Italian, ancient Greek, noise) and gap length ( 3 or $7 \mathrm{sec}$ ) as factors showed a significant effect of materials, as was expected $[\mathrm{F}(2,38)=6.65, p<.01]$. For 3-sec gaps, pauses inserted in Italian question-answer pairs were estimated to be $4.2 \mathrm{sec}$, versus $3.9 \mathrm{sec}$ for the Greek material and $3.6 \mathrm{sec}$ for noise. For 7-sec gaps, pauses inserted in the Italian and Greek material were estimated to be $7.2 \mathrm{sec}$, versus $6.6 \mathrm{sec}$ for noise.

A second ANOVA for the Italian data only, including knowledge domain (autobiographical vs. general), difficulty (easy vs. hard), and gap length as factors, showed a significant effect of difficulty $[F(1,19)=9.29, p<.01]$. Three- and 7-sec gaps left following an easy question were estimated as being 4.3 and $7.4 \mathrm{sec}$ in length, versus 4.2 and $7.0 \mathrm{sec}$ for difficult questions.

\section{EXPERIMENT 2}

\section{Method}

Stimulus material. The stimulus material was a set of 24 experimental and filler questions. The results reported here concern eight questions defined as in Experiment 1 and varying on two dimensions: difficulty (easy vs. hard) and knowledge domain (autobiographical vs. general). The remaining 16 items included 8 questions judged as being of medium difficulty and 8 questions included as foils.

Subjects, Task, and Procedure. The twelve university students tested conducted an interview by telephone. The subjects were told that their task was to gather certain information and that they could do this by telephoning a person who had previously agreed to be interviewed. Each subject was to ask the person interviewed the full set of 24 questions (prepared for each subject in a different random order). The person interviewed, a confederate of the experimenter but unaware of the study's purpose, received instructions not to answer 4 of the 8 critical questions (counterbalanced over subjects) until the speaker repeated it or made another conversational move (e.g., "Do you hear me?"; Are you still there?'). For half of these questions, the delay in answering was to be completely mute; for the other half, it was to include, after a 1-sec mute delay, a sound ("mm...") serving as a simple feedback signal. This use of feedback defined a third experimental factor.

\section{Results}

Table 1 summarizes the elapsed time between the offset of each unanswered question and the onset of a further solicitation or prompting of an answer. An ANOVA performed on these data, including difficulty (easy vs. hard), knowledge domain (autobiographical vs. general), and feedback (none vs. vocal gesture) as factors, yielded a significant main effect only for difficulty $[F(1,11)=16.27, p<.001]$, with pauses of $16 \mathrm{sec}$ tolerated for hard questions versus $13 \mathrm{sec}$ for easy ones.
Table 1

Mean Gap (in seconds) Left Before Speakers Took an Additional Turn in Experiment 2

\begin{tabular}{|c|c|c|c|c|}
\hline \multirow{3}{*}{$\begin{array}{c}\text { Feedback } \\
\text { Signal Used }\end{array}$} & \multicolumn{4}{|c|}{ Knowledge Type } \\
\hline & \multicolumn{2}{|c|}{ Autobiographical } & \multicolumn{2}{|c|}{ General } \\
\hline & Easy & Difficult & Easy & Difficult \\
\hline yes & 12.9 & 19.75 & 13.9 & 15.4 \\
\hline no & 11.25 & 16.0 & 14.25 & 14.1 \\
\hline
\end{tabular}

An interaction between difficulty and knowledge domain $[F(1,11)=5.15, p<.05]$, however, confirmed that the effect was limited to autobiographical questions (12 sec tolerated for easy questions, $18 \mathrm{sec}$ for difficult). Third, the interaction of difficulty and use of feedback approached significance $[F(1,11)=4.12, .05<p<.1]$, with feedback making a difference only for hard questions (14 sec tolerated without feedback, $18 \mathrm{sec}$ with feedback).

\section{GENERAL DISCUSSION}

The present research was designed with the aim of contributing experimental data to a grammar of conversation. Our goal was to describe and define the mutual interaction between temporal patterns of turntaking and cognitive processes, also with reference to "naive" psychological theories ("thinking takes time"). We hypothesized that the "empty" parts in conversational exchanges are functional constituents in a dialogue (see also Beattie \& Barnard, 1979; Jaffe \& Feldstein, 1970; Wilson \& Zimmermann, 1986), and that, other things being equal, a gap acquires its value and meaning according to its duration and position in the flow of discourse.

Experiment 1 showed that the understanding and complexity of question-answer exchanges that are overheard influences the perception of pause separating them. Experiment 2 cast light on a speaker's tolerance for, or interruption of, a listener's silence. The results stress the existence of, and a subject's adherence to, an implicit temporal rule in conversation, which states that an excessive gap will be perceived as deviant and corrected. But, second, the same phenomenon points to the necessity of a description of turn-taking rules also in terms of processes of inference. Partly, these concern the amount of knowledge necessarily activated before beginning a turn, and the inner mental processes attributed to the person who is the next invited speaker.

Previous research on language production offers a consistent interpretation of intraturn gaps in terms of time actually required by lexical or syntactic decisions. Interturn gaps, on the other hand, have usually been studied and explained within a more general model of alternation in conversational turns, which stresses the temporal rhythm of discourse but underestimates the relevance of discourse content. The particular case of the adjacent question-answer pair in a dyadic conversation rules out the problem of selecting the next speaker and at the same time allows us to focus on mechanisms of maintenance of the conversational interaction, while taking into account the complexity of mental work required. The gist of the rule underlying gap length, according to the results obtained in our experiments, is that the duration of a gap, and the extent to which it is accepted or tolerated by a conversational partner, depends on the kind of question asked-that is, on characteristics of the question content. Furthermore, the rule states that, if a question is not answered within a maximal delay appropriate for its content, a signal of question reception is needed if a disruption of the interaction is to be avoided.

\section{REFERENCES}

Beattie, G. W., \& BARNARD, P. (1979). The temporal structure of natural telephone conversation. Linguistica, 17, 213-230.

BUTTERWORTH, B. (1980). Evidence from pauses in speech. In 
B. Butterworth (Ed.), Language production: Speech and talk (Vol. 1, pp. 155-176). London: Academic Pess.

FraIsSE, P. (1978). Time and rhythm perception. In E. C. Carterette \& M. P. Friedman (Eds.), Handbook of perception (Vol. 8, pp. 203254). New York: Academic Press.

Goldman-Eisler, F. (1968). Psycholinguistics: Experiments in spontaneous speech. New York: Academic Press.
JAFFE, J., \& FeldSteIn, S. (1970). Rhythms of dialogue. New York: Academic Press.

Wilson, T. P., \& Zimmermann, D. H. (1986). The structure of silence between turns in two-party conversation. Discourse Processes, 9, 375-390.

(Manuscript received May 25, 1989.) 